Note

\title{
Measurement of the Rate of Myofibrillar Protein Degradation Using the Arteriovenous Difference in Plasma 3-Methylhistidine Concentration of Rats
}

\author{
Takayuki SugaWARA ${ }^{1}$, Yoshiaki ITO' ${ }^{2}$, Naoyuki NisHIZAWA ${ }^{2}$, Hiromi SUZUKI ${ }^{3}$, \\ Hisamine KOBAYASHI ${ }^{3}$ and Takashi NAGASAWA ${ }^{2, *}$ \\ ${ }^{1}$ United Graduate School of Agricultural Sciences, Iwate University, Morioka, Iwate 020-8550, Japan \\ ${ }^{2}$ Depatment of Biological Chemistry and Food Sciences, Faculty of Agriculture, Iwate University, \\ Morioka, Iwate 020-8550, Japan \\ ${ }^{3}$ AminoScience Laboratories, Ajinomoto Co., Inc., Kawasaki, Kanagawa 210-8681, Japan
}

(Received January 23, 2009)

\begin{abstract}
Summary There are several methods for measuring the rate of myofibrillar protein degradation using 3-methylhistidine (3-MeHis) levels in urine, plasma and isolated muscle. However, these methods lack the accuracy of rate measurements. Therefore, it is necessary to develop a new method for determining the rate of myofibrillar protein degradation. We characterized an arteriovenous difference method using 3-MeHis plasma concentration. Rats were fasted overnight and subsequently administered leucine $(135 \mathrm{mg} / 100 \mathrm{~g} \mathrm{BW})$ or fed a $20 \%$ casein diet. The rate of myofibrillar protein degradation was calculated by multiplying the femoral artery blood flow rate by the arteriovenous difference in 3-MeHis concentrations (vein-artery). The blood was collected from the femoral vein and abdominal aorta. The release of 3-MeHis from hindlimb muscle was significantly suppressed $(p<0.05)$ in rats fed leucine or the $20 \%$ casein diet, indicating that myofibrillar protein degradation was suppressed. These results suggest that the evaluation of the rate of myofibrillar protein degradation using the arteriovenous difference method reflects nutritional conditions in a more physiological manner.
\end{abstract}

Key Words arteriovenous difference, 3-methylhistidine, myofibrillar protein degradation, leucine

Sarcopenia, where the synthesis of muscle protein is reduced and degradation is increased, can occur as a result of catabolic diseases and aging (1). Therefore, in order to increase or maintain muscle mass, it is important to regulate muscle protein synthesis and degradation. The accurate measurement of the rate of muscle protein synthesis and degradation is necessary to understand the regulation of muscle mass. Methods for measuring the rate of protein synthesis generally involve the incorporation of labeled amino acids into proteins or assessment of the kinetics of labeled amino acid distribution between tissue and blood. However, methods for measuring protein degradation have not been fully validated. Myofibrils which are the main component of skeletal muscle, are mainly composed of actin and myosin, and function in muscle contraction. Therefore, accurate measurement of the rate of myofibrillar protein degradation is meaningful for evaluating the regulation of muscle protein degradation.

3-Methylhistidine ( $N^{\tau}$-methylhistidine (3-MeHis)) is localized in actin and myosin, and is not reused for protein synthesis (2). Urinary excretion of 3-MeHis is used

\footnotetext{
*To whom correspondence should be addressed.
}

E-mail: tnaga@iwate-u.ac.jp as an index of the rate of myofibrillar protein degradation (3). However, urinary 3-MeHis does not appear to reflect myofibrillar protein degradation of skeletal muscle alone, because 3-MeHis is also released from tissues other than skeletal muscle. Furthermore, this method cannot measure acute changes in the rate of muscle protein degradation as the collection period for urine is typically over $24 \mathrm{~h}$. On the other hand, plasma 3-MeHis concentration is also used as an index of the rate of myofibrillar protein degradation, and can reflect acute changes in the degradation rate (4). However, plasma 3-MeHis is not limited to skeletal muscle origin, and may be affected by kidney function or blood flow. The release of 3-MeHis from isolated muscle can directly measure the rate of myofibrillar protein degradation (5, 6 ). However, the isolated muscle must be thin, such as the extensor digitorum longus (EDL) or soleus muscle, and weigh less than $50 \mathrm{mg}(1,6,7)$. Therefore, these criteria limit studies to those using rats with a body weight of less than $100 \mathrm{~g}$. Furthermore, this method may not be physiological, because the isolated muscle is no longer regulated by the nervous system and hormonal action. Thus, it is necessary to use a more accurate and physiological method to measure myofibrillar protein degradation. 
A

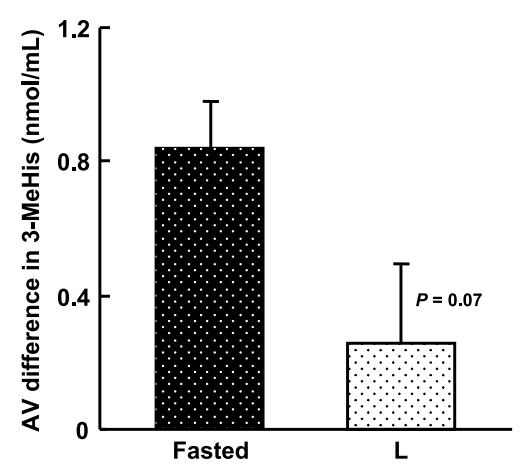

B

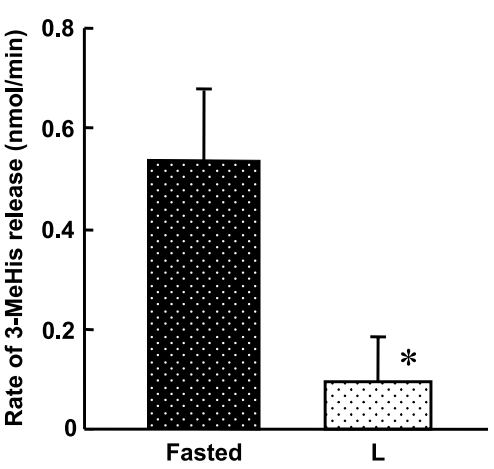

Fig. 1. Effect of oral administration of leucine post-fasting on the AV difference in 3-MeHis concentrations (A) and the rate of myofibrillar protein degradation in young rats (B) $(80 \mathrm{~g} \mathrm{BW})$. Values are mean \pm SE $(n=5) .{ }^{*} p<0.05$ versus Fasted group. Fasted, fasted for $24 \mathrm{~h}$; L, administered leucine (135 mg/100 g BW).

The aim of the present study was to find a new method to measure the rate of myofibrillar protein degradation. In this study, we applied an arteriovenous (AV) concentration difference technique (8) using plasma 3-MeHis concentrations. Our results indicated that utilization of the AV difference method enabled the use of 3-MeHis concentrations to detect differences in the nutritional status in rats.

\section{Materials and Methods}

Animals and diet.

Experiment 1: Male Wistar rats (4 wk old) were purchased from Japan SLC, Inc. (Shizuoka, Japan). They were individually housed in stainless steel wire cages and maintained at $22^{\circ} \mathrm{C}$ and $55 \%$ relative humidity with a 12-h light dark cycle (6:00-18:00). They were allowed free access to tap water and the AIN-93G 20\% casein diet (20C) (9). The animal care protocol in this study was approved by the Iwate University Animal Research Committee under Guidelines for Animal Experiments in Iwate University.

When the weight of the rats reached approximately $80 \mathrm{~g}$ ( $5 \mathrm{wk}$ old), the rats were randomly assigned to 2 groups ( $n=5$ in each group) and fasted for $24 \mathrm{~h}$. The rats of one group (L, leucine) were administered leucine (135 mg/100 g BW by a feeding tube (Safeed Fr. 5, Termo Co., Tokyo, Japan), and the rate of myofibrillar protein degradation measured $3 \mathrm{~h}$ after administration. Myofibrillar protein degradation was also measured in the remaining group (Fasted) after fasting alone.

Experiment 2: Male Wistar rats ( 7 wk old) were purchased from Japan SLC, Inc. They were allowed free access to tap water and the $20 \%$ casein diet. When the weights of the rats reached approximately $200 \mathrm{~g} \mathrm{(9} \mathrm{wk}$ old), the rats were randomly assigned to 3 groups $(n=5$ in each group), and fasted for $18 \mathrm{~h}$. The first group (20C) was fed the $20 \%$ casein diet for $1 \mathrm{~h}$, and the rate of myofibrillar protein degradation measured $4 \mathrm{~h}$ after refeeding. The second group (L) was administered leucine $(135 \mathrm{mg} / 100 \mathrm{~g} \mathrm{BW})$ and the rate of myofibrillar protein degradation measured $3 \mathrm{~h}$ after administration. The rate of myofibrillar protein degradation was measured in the third group (Fasted) after fasting alone.
Measurement of myofibrillar protein degradation. To measure the rate of myofibrillar protein degradation we employed the AV concentration difference method (8). The rats were anesthetized with intraperitoneal injections $(188 \mu \mathrm{L} / 100 \mathrm{~g} \mathrm{BW})$ of a mixture of ketamine $(43.1 \mathrm{mg} / \mathrm{mL})$ and xylazine $(2.76 \mathrm{mg} / \mathrm{mL})$. The epigastric vein and artery were then banded with surgical sutures (Natume, Tokyo, Japan). To measure the blood flow rate, a transonic-flow probe (1PRB 2972, Transonic Systems Inc, Ithaca, NY, USA) was positioned around the femoral artery, and the flow rate monitored using a transit-time flowmeter (TS420, Transonic Systems Inc). Blood was then collected from both the femoral vein and abdominal aorta. The rate of myofibrillar protein degradation was calculated using the following equation.

$$
Q=\left(C_{V}-C_{A}\right) \times F
$$

where $Q$ is the rate of myofibrillar protein degradation (release of 3-MeHis from hindlimb muscle, $\mathrm{nmol} / \mathrm{min}$ ), $C_{\mathrm{A}}$ is the arterial blood 3-MeHis concentration (nmol/ $\mathrm{mL}), C_{V}$ is that in venous blood $(\mathrm{nmol} / \mathrm{mL})$, and $F$ is the blood flow rate $(\mathrm{mL} / \mathrm{min})$.

The blood was centrifuged at 3,500 $\times g$ for $15 \mathrm{~min}$ to obtain plasma, which was frozen in liquid nitrogen and stored at $-80^{\circ} \mathrm{C}$ until analysis. Plasma 3-MeHis concentrations were measured by HPLC after derivatization with o-phthalaldehyde according to Nagasawa and coworkers (10) with a small modification for volume of $o$-phthalaldehyde solution.

Statistical analyses. Data are expressed as mean \pm SE. Comparisons between the fasted groups and other groups were carried out using a two-tailed unpaired $t$ test (Instat Ver. 2.03). Differences were considered significant at $p<0.05$.

\section{Results and Discussion}

It is important to accurately measure the rate of muscle protein degradation in order to understand the loss of muscle mass during aging and disease. We first measured the rate of myofibrillar protein degradation by the 3-MeHis AV concentration difference in young rats (Experiment 1). The blood flow rate was significantly decreased $(p<0.05)$ by oral administration of leucine 


\section{A}

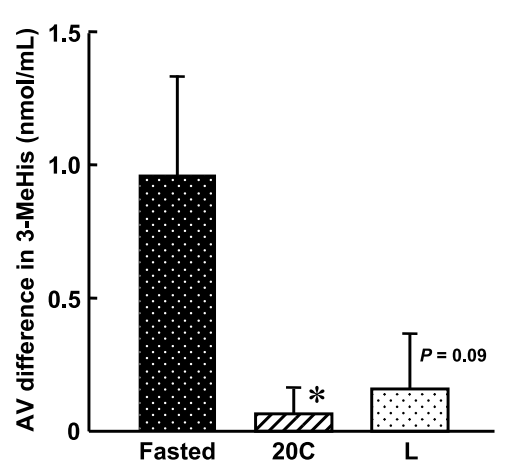

B

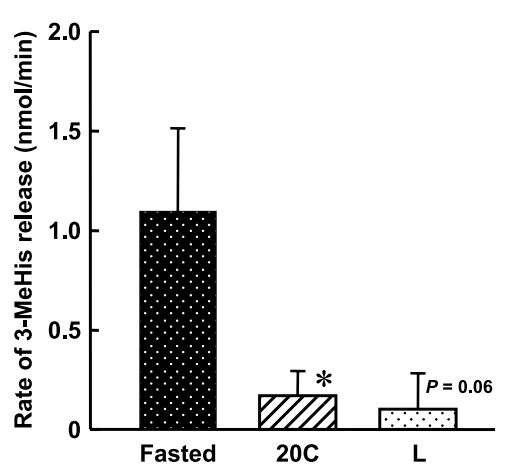

Fig. 2. Effect of feeding a 20\% casein diet or administration of leucine post-fasting on the AV difference in 3-MeHis concentrations (A) and the rate of myofibrillar protein degradation in adult rats (B) $(200 \mathrm{~g} \mathrm{BW})$. Values are mean \pm SE $(n=5)$. ${ }^{*} p<0.05$ versus Fasted group. Fasted, fasted for 18 h; 20C, refed 20\% casein diet; L, administered leucine (135 mg/100 g $\mathrm{BW})$.

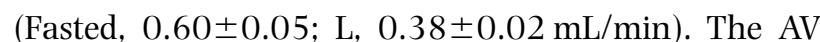
difference in 3-MeHis concentration (vein-artery) tended to decrease when rats were orally administered leucine (Fig. 1A). The rate of 3-MeHis release (flow rate $\times 3-\mathrm{MeHis} \mathrm{AV}$ difference) from the hindlimb muscle in rats administered leucine was significantly suppressed $(p<0.05 ; 82 \%)$ as compared to the Fasted group. This result suggests that the oral administration of leucine dramatically suppressed myofibrillar protein degradation (Fig. 1B). In our previous study where the rate of myofibrillar protein degradation was measured by the release of 3-MeHis from isolated EDL muscle, the rate was also suppressed by oral administration of leucine (6). The consistency with our previous results strongly suggests that the method used herein to measure the 3-MeHis AV difference can be successfully applied to estimate the rate of myofibrillar protein degradation.

In Experiment 2, we measured the rate of myofibrillar protein degradation using the plasma 3-MeHis AV difference in rats weighing $200 \mathrm{~g}$. The blood flow rate tended to decrease following administration of leucine ( $p=0.09$; Fasted, 1.12 $\pm 0.13 ; 20 \mathrm{C}, 0.92 \pm 0.04 ; \mathrm{L}$, $0.78 \pm 0.12 \mathrm{~mL} / \mathrm{min})$. The $3-\mathrm{MeHis} \mathrm{AV}$ difference was significantly decreased $(p<0.05)$ by refeeding of the $20 \%$ casein diet and tended to decrease with the leucine administration (Fig. 2A). The rates of 3-MeHis release from hindlimb muscles of rats fed the $20 \%$ casein diet and administered leucine was suppressed by $85 \%$ $(p<0.05)$ and 91\% $(p=0.06)$, respectively, as compared to the Fasted group (Fig. 2B). Therefore, the feeding of dietary casein or leucine suppressed myofibrillar protein degradation, not only in young rats but also in adult rats. In our previous studies, we suggested that the feeding of dietary casein or leucine can suppress myofibrillar protein degradation in young rats, which was evaluated by the release of 3 -MeHis from isolated muscle (5, 6 ). However, to date, it was unknown whether the effect of feeding of dietary casein or leucine on muscle protein degradation in larger rats over $100 \mathrm{~g} \mathrm{BW}$, as evaluated by 3-MeHis release, is similar to the effect observed in young rats, because the method of using isolated mus- cle cannot be used with the tissue from the large rats. On the other hand, the use of the 3-MeHis AV difference method is not limited by animal body weight as demonstrated herein; we were able to measure the rate of myofibrillar protein degradation independently of rat body weight.

Vesali et al. (11) suggested that the AV difference method produced results with excessive variability in the plasma 3-MeHis concentrations when measured by HPLC. Therefore, they recommended that the 3-MeHis AV difference should be measured by the isotope dilution method. Certainly, plasma 3-MeHis concentrations are very low. However, in the present study we were able to demonstrate 3-MeHis AV differences as measured by a highly sensitive HPLC method (10); and as a result, the difference accurately reflected the animal's nutritional status. Kobayashi et al. (12) measured muscle protein degradation using the 3-pool model, which can evaluate the rate of degradation as well as synthesis. However, this method requires the use of labeled amino acids. The 3-MeHis AV difference method does not require labeled amino acids and can directly measure the rate of myofibrillar protein degradation.

In conclusion, we have demonstrated that 3 -MeHis AV concentration differences can measure the rate of myofibrillar protein degradation in the rat model. This method provides a more physiological evaluation (directly and accurately) of the rate of myofibrillar protein degradation, independent of rat body weight.

\section{Acknowledgments}

The authors thank Dr. Yvonne Yuan, Ryerson University, Toronto, Canada for helpful discussion. This work was supported by a Grant-in-Aid for Scientific Research (C) from the Ministry of Education, Culture, Sports, Science and Technology of Japan.

\section{REFERENCES}

1) Hasselgren PO, James JH, Benson DW, Hall-Angeras M, Angeras U, Hiyama DT, Li S, Fischer JE. 1989. Total and myofibrillar protein breakdown in different types of rat skeletal muscle: effects of sepsis and regulation by insu- 
lin. Metabolism 38: 634-640.

2) Young VR, Munro HN. 1978. $N^{\tau}$-methylhistidine (3methylhistidine) and muscle protein turnover: an overview. Fed Proc 37: 2291-2300.

3) Haverberg LN, Deckelbaum L, Bilmazes C, Munro HN, Young VR. 1975. Myofibrillar protein turnover and urinary $N^{\tau}$-methylhistidine output. Response to dietary supply of protein and energy. Biochem J 152: 503-510.

4) Nagasawa T, Yoshizawa F, Nishizawa N. 1996. Plasma $N^{\tau}$-methylhistidine concentration is a sensitive index of myofibrillar protein degradation during starvation in rats. Biosci Biotechnol Biochem 60: 501-502.

5) Nagasawa T, Hirano J, Yoshizawa F, Nishizawa N. 1998. Myofibrillar protein catabolism is rapidly suppressed following protein feeding. Biosci Biotechnol Biochem 62: 1932-1937.

6) Nagasawa T, Kido T, Yoshizawa F, Ito Y, Nishizawa N. 2002. Rapid suppression of protein degradation in skeletal muscle after oral feeding of leucine in rats. J Nutr Biochem 13: 121-127.

7) Nagasawa T, Kikuchi N, Ito Y, Yoshizawa F, Nishizawa N. 2004. Suppression of myofibrillar protein degradation after refeeding in young and adult mice. J Nutr Sci Vita- minol 50: 227-230.

8) Macdonald IA. 1999. Arterio-venous differences to study macronutrient metabolism: introduction and overview. Proc Nutr Soc 58: 871-875.

9) Reeves PG, Nielsen FH, Fahey GC Jr. 1993. AIN-93 purified diets for laboratory rodents: final report of the American Institute of Nutrition ad hoc writing committee on the reformulation of the AIN-76A rodent diet. J Nutr 123: 1939-1951.

10) Nagasawa T, Sakai T, Onodera R. 1991. Simple and sensitive determination of plasma $N^{\tau}$-methylhistidine by high-performance liquid chromatography using precolumn derivative formation with $o$-phthalaldehyde-2mercaptoethanol. J Chromatogr 566: 223-227.

11) Vesali RF, Klaude M, Thunblad L, Rooyackers OE, Wernerman J. 2004. Contractile protein breakdown in human leg skeletal muscle as estimated by $\left[{ }^{2} \mathrm{H}_{3}\right]-3-$ methylhistidine: a new method. Metabolism 53: $1076-$ 1080.

12) Kobayashi H, Kato H, Hirabayashi $Y$, Murakami H, Suzuki H. 2006. Modulations of muscle protein metabolism by branched-chain amino acids in normal and muscle-atrophying rats. J Nutr 136: 234S-236S. 